\title{
Application of exogenous xyloglucan oligosaccharides affects molecular responses to salt stress in Arabidopsis thaliana seedlings.
}

\author{
Lien González-Pérez ${ }^{1 *}$, Timothy Páez-Watson², José M. Álvarez-Suarez², Mayra C. \\ Obando-Rojas ${ }^{2}$, Edison Bonifaz-Arcos ${ }^{2}$, Gabriela Viteri², Fernando Rivas-Romero², \\ Eduardo Tejera $^{1}$, Hilary J. Rogers ${ }^{3}$, Juan C. Cabrera ${ }^{4,5}$
}

${ }^{1}$ Facultad de Ciencias de la Salud, Universidad de las Américas (UDLA), Sede UDLAPARK vía a Nayón. CP. 170513, Quito, Ecuador. ${ }^{2}$ Facultad de Ingeniería y Ciencias Aplicadas (FICA), Universidad de las Américas (UDLA), Calle José Queri s/n entre Av. Granados y Av. Eloy Alfaro. CP. 170513, Quito, Ecuador.

${ }^{3}$ School of Biosciences, Cardiff University, Sir Martin Evans Building, Park Place, Cardiff, CF10 3AX, United Kingdom.

${ }^{4}$ Unité de Biotechnologie, Materia Nova, Rue des Foudriers, 1, 7822 Ghislenghien, Belgium.

${ }^{5}$ Fyteko sprl. Brussels, Belgium. *Corresponding author: liengonza@yahoo.es

\begin{abstract}
Soil salinity is one of the most devastating problems which reduces crop production and increases desertification. New approaches to overcome the negative effect of salinity on plants include the use of plant biostimulants, such as Xyloglucan oligosaccharides (XGOs) derived from the breakdown of xyloglucans from plant cell walls. The present study aimed at verifying the influence of exogenous XGOs derived from Tamarindus indica L. cell walls, on Arabidopsis thaliana's tolerance to salt stress by understanding the gene expression, enzymatic and metabolic changes resulting from its application. A. thaliana plants were grown in liquid media and after 15 days they were treated by a salt shock with $100 \mathrm{mM}$ of sodium chloride, with or without XGOs at $0.1 \mathrm{mg} \mathrm{L}^{-1}$. Gene expression of four oxidative stress markers as well as catalase and peroxidase activities and content of glutathione, total carbonyl, polyphenolics and chlorophyll were quantified. Bioinformatic models were used to obtain the co-expression network of the four oxidative stress response gene markers from microarray data of Arabidopsis under salt stress. Results showed that in saline conditions, XGOs dramatically increased catalase gene expression and enzymatic activity, peroxidase activity, and chlorophyll $\mathrm{a} / b$ ratio, while reducing protein oxidation and total polyphenols. Thus, XGOs may act to counteract negative effects of oxidative stress under saline conditions.
\end{abstract}

Keywords: Arabidopsis thaliana, antioxidative response, salinity stress, xyloglucan oligosaccharides. 


\section{Introduction}

Among the various environmental stresses, salinity in soil or water is one of the most devastating problems affecting commercial crop production. Soils affected by salinity are those where the electrical conductivity exceeds $4 \mathrm{dSm}^{-1}(\approx 40 \mathrm{mM}$ sodium chloride) and also contain excessive soluble salts. The most abundant, soluble, persistent and detrimental salt in saline soils worldwide is sodium chloride $(\mathrm{NaCl})$. Consequently, salt stress is induced mainly by $\mathrm{Na}^{+}$and $\mathrm{Cl}^{-}$ions which can be transported into and out of cells (Abogadallah, 2010).

Plants react to salt stress by inducing numerous mechanisms. Adverse consequences of salt stress include effects on seed germination, plant growth and development, enzymatic activity and disruption of photosynthesis (Abogadallah, 2010), the latter being one of the most important effects. The photosynthetic response to salt stress is highly complex, but the most relevant feature of this process includes a reduction in the concentration of chlorophyll $(\mathrm{Chl})$ and damage to the photosynthetic apparatus. Chloroplasts possess up to $70 \%$ of total leaf proteins and their degradation can alter the photosynthetic capacity of plants and consequently crop yield. Recently, expression of a nuclear-encoded Arabidopsis gene for chloroplast vesiculation $(\mathrm{CV})$ under abiotic stress was reported, specifically under salt stress with $\mathrm{NaCl}$. Its function is to target and destabilize chloroplasts for protein degradation and induce the formation of vesicles enclosing thylakoid proteins (Wang and Blumwald 2014)

Another consequence of saline growth condition is the generation of oxidative stress at the cellular level, characterized by the presence of transitorily active reactive oxygen species (ROS), such as the hydroxyl radical, superoxide radical and singlet oxy- gen (Abogadallah 2010). In plants, these molecules are formed due to the incomplete reduction of molecular oxygen caused by the energy flow during electron transfer. ROS are produced continuously, principally in chloroplasts, mitochondria and peroxisomes, and are induced by various stresses at all phases of development (You and Chan 2015). Overproduction of ROS is highly detrimental and results in cellular damage, including membrane destruction, lipid peroxidation, pigment oxidation, protein denaturation and DNA mutation, which ultimately results in cell death. Plants possess efficient enzymatic and non-enzymatic antioxidative systems to avoid oxidative damage and maintain non-toxic levels of ROS Several ROS-scavenging enzymes in plants work together to detoxify ROS, including catalase (CAT), ascorbate peroxidase (APX), glutathione reductase (GR) and peroxiredoxin (Prx) (You and Chan 2015). Different strategies in agricultural practices have been developed to overcome the negative effect of salinity in plants, increase nutrient uptake and enhance crop performance. Some organic molecules are able to activate plant metabolism and improve their performance under different kind of stresses in a short period of time and inexpensively (Bulgari et al., 2015). New approaches include the use of plant biostimulants, bioactive compounds that operate through different mechanisms to fertilizers. Biostimulants are usually able to enhance tolerance to biotic and abiotic stresses in plants and improve their nutrient use efficiency which in consequence, allow a reduction in fertilizers without affecting yield and quality (Bulgari et al., 2015). However, it is not possible to suggest a common mode of action for all formulations because they derive from multiple sources or contain a variety of bioactive components which together, may contribute to specific effects in plants. 
Xyloglucan oligosaccharides (XGOs), derived from the breakdown of xyloglucans, are gaining importance in agriculture as well as in their use as functional food ingredients, in the pharmaceutical industry, and feed formulations (Kozioł et al., 2015). Xyloglucan is found in all cell walls of all vascular plants and is the major hemicellulose polysaccharide in the primary cell wall of eudicots and non-graminaceous monocots. Xyloglucan consists of a $\beta$ - $(1 \rightarrow 4)$-glucan backbone substituted with $\alpha-(1 \rightarrow 6)$-xylosyl residues in a regular pattern, as well as occasional galactosyl or fucosyl residues (Kozioł et al., 2015). It is a valuable source for natural polysaccharides and oligosaccharides with specific biological activity (Cabrera et al., 2012).

XGOs from tamarind (Tamarindus indica L.) seeds showed biological activity at concentrations of 0.1 $\mathrm{mg} \mathrm{L}^{-1}$ in Nicotiana tabacum and Arabidopsis thaliana (Cabrera et al., 2012). These oligosaccharides are thus characterized by their activity as biostimulants at very low concentration. XGOs also had a significant effect on plant growth and on the expression of genes related to cell signaling, metabolism, division, transcriptional control and stress response in tobacco BY-2 cells (González-Pérez et al., 2014). One notable finding from the microarray analysis of tobacco BY-2 cells was the large number of stress responsive genes that were differentially expressed. This suggests a link between XGO treatment and abiotic stress protection, which may be of relevance for their use as plant biostimulants and as part of the mechanism of XGO action. Despite the fact that XGOs are emerging as a new class of naturally occurring biostimulants, to our knowledge there are no previous studies on plant gene expression, protein concentrations, enzyme activity or metabolic responses related to oxidative stress mediated by XGOs under saline conditions.

The present work was focused on assessing the effects of XGOs on A. thaliana's response to salt shock by analyzing gene-expression, enzymatic and metabolic changes. The overall aim was to gain a better understanding of their mechanism of action in support of their application as plant biostimulants.

\section{Materials and Methods}

\subsection{Xyloglucan Oligosaccharides $(X G O)$}

Xyloglucan (XG) polysaccharide was extracted from tamarind (Tamarindus indica L.) seeds and purified as described previously (Cutillas-Iturralde et al., 1998). Trichoderma viride cellulase (Sigma) was used to digest the XG polysaccharide, and the xyloglucan oligosaccharides (XGOs) produced were isolated by ultrafiltration (Amicon centrifugal filter devices MWcut off 5,000 Da). The retentate fraction (containing the enzyme and undegraded polysaccharides) was discarded and the permeate fraction containing the XGOs was exhaustively dialyzed against deionized water (Spectra/Por MWcut off $500 \mathrm{Da}$ ) and freeze dried. The isolated XGO fraction showed no cellulase activity and protein could not be detected. The uniformity of the XGO mixture was confirmed by gel filtration analysis through BioGel P2 (Bio-Rad Laboratories, Inc., Hercules, CA, USA). XGO composition was assessed using Matrix Assisted Laser Desorption Ionisation-Time of Flight (MALDI-TOF) spectrometry (Mazumder et al., 2005). Mass spectra revealed XGO ions with $\mathrm{m} / \mathrm{z}$ of $791,953,1085,1247$ and 1409 corresponding to $(\mathrm{M}+\mathrm{Na})+$ adduct ions of XXG, XXGG, XXXG, $\mathrm{XXLG/XLXG} \mathrm{(XGO} \mathrm{isomers} \mathrm{are} \mathrm{not} \mathrm{discriminated}$ by MALDI-TOF analysis), and XLLG respectivelly (Table 1). The XGO mixture was chiefly XLLG and XXLG, with a lower proportion of XXXG XXGG and XXG, as classified by Fry et al, (1993). Relative proportions of xyloglucan oligosaccharides obtained by MALDI and HAEC-PAD analysis were similar (data not show). 
Table 1. XGO composition in cellulase hydrolysates of Tamarindus indica L. xyloglucan as determined my MALDI-TOF mass spectrometry.

\begin{tabular}{|c|c|c|c|}
\hline $\begin{array}{c}(\mathrm{M}+\mathrm{Na})^{+} \\
\mathrm{m} / \mathrm{z}\end{array}$ & $\begin{array}{l}\text { Number of glucosyl } \\
\text { residues }^{\mathrm{a}}\end{array}$ & Assigned structure $^{b}$ & $\begin{array}{c}\text { Relative proportion } \\
(\%)\end{array}$ \\
\hline 791 & Hex $_{3}$ Pent $_{2}$ & $\mathrm{XXG}$ & 3 \\
\hline 953 & $\mathrm{Hex}_{4} \mathrm{Pent}_{2}$ & XXGG & 7 \\
\hline 1085 & $\mathrm{Hex}_{4} \mathrm{Pent}_{3}$ & XXXG & 16 \\
\hline 1247 & $\mathrm{Hex}_{5} \mathrm{Pent}_{3}$ & XXLG/XLXG & 36 \\
\hline 1409 & $\mathrm{Hex}_{6} \mathrm{Pent}_{3}$ & XLLG & 33 \\
\hline
\end{tabular}

\footnotetext{
${ }^{a}$ Number of glucosyl residues: The deduced number of hexosyl (Hex) and pentosyl (Pent) residues in each oligosaccharide

${ }^{\mathrm{b}}$ Nomenclature according Fry et al. (1993).
}

\subsection{Plant materials and growth conditions}

Seeds of $A$. thaliana (L.) Heynh, ecotype Col-0, were surface sterilized and grown in $100 \mathrm{~mL}$ of Murashige and Skoog (MS) liquid media (Murashige and Skoog 1962), in a growth chamber (DAIHAN Scientific, model WISD, Korea) at $23{ }^{\circ} \mathrm{C}$ in long-day conditions (16 h light/ $8 \mathrm{~h}$ dark), at $50 \%$ relative humidity and $200 \mathrm{rpm}$ for 15 days. After that time, the exhausted medium was discarded and replaced with fresh MS medium and plants were maintained for 24 hours under the same conditions. This step was performed to reduce any stress that might occur as a result of the change of medium. Subsequently, leaf samples were taken at Time $0\left(\mathrm{~T}_{0}\right)$, which served as a negative control for all analyses. Remaining plants were immediately transferred into fresh media containing $0.1 \mathrm{mg} \mathrm{L}^{-1} \mathrm{XGO}$ (corresponding to $0.1 \mu \mathrm{M}$ ) or 100 $\mathrm{mM}$ sodium chloride to induce a salt shock, as an intermediate salt level (Shavrukov 2013) or a combination of both $(\mathrm{XGO}+\mathrm{NaCl})$. The concentration of $\mathrm{XGO}$ was selected based on previous experiments as an optimal concentration for eliciting plant responses. In Arabidopsis thaliana, XGOs were previously evaluated from 0.005 to $10 \mathrm{mg} \mathrm{L}^{-1}$; however, higher concentrations exhibited an inhibitory effect on seed germination, root development and plant growth. The optimal XGO concentration for eliciting an increase in primary root elongation and leaf development was $0.1 \mathrm{mg} \mathrm{L}^{-1}$ (González-Pérez et al., 2014; GonzálezPérez et al., 2012; Cabrera et al., 2012).

According to previous studies, salt shock is an extreme form of salt stress and after 24-72 hours of $\mathrm{NaCl}$ application, the concentration of $\mathrm{Na}^{+}$in the cytoplasm may be close to toxic levels (Shavrukov 2013). Thus, plants were grown in each solution (with or without salt) from 24 to 72 hours after induction. Leaf samples were taken at time intervals and were 
immediately frozen in liquid nitrogen and stored at $-80{ }^{\circ} \mathrm{C}$ until used. Each experiment was repeated to generate three biological replicates.

\subsection{RNA extraction and quantitative RT-PCR}

Total RNA was obtained from $100 \mathrm{mg}$ of plant leaves collected at times 0, 24 and 72 hours after induction using a PureLink RNA mini kit (Ambion, Life technologies, USA) and following the manufacturer's instructions. Residual DNA was removed by treatment of the extracted total RNA with RNase free DNase I (Ambion, Life technologies, USA) and verified by $1 \%$ agarose gel electrophoresis. RNA concentration was measured by spectrophotometry (BMG LABTECH GmbH, model FLUOstar Omega, Germany). To perform the quantitative reverse transcription and polymerase chain reaction (qRT-PCR) assays, aliquots of RNA were prepared by dilution of the obtained product to a final concentration of $300 \mathrm{ng} \mu \mathrm{L}^{-1}$. Gene specific primers for A. thaliana were designed from the annotated sequences reported in the TAIR database (The Arabidopsis Information Resource, https://www.arabidopsis.org/) and using the program Primer3 (http://bioinfo.ut.ee/ primer3-0.4.0/). Gene specific primers were designed for amplification of catalase, based on a conserved region of catalase 2 and 3 in order to detect both isoforms simultaneously (EC 1.11.1.6, CAT, At1g20620), L-Ascorbate Peroxidase 1 (EC 1.11.1.1, APX, At1g07890), Peroxiredoxin $Q$ (EC 1.11.1.15, $\operatorname{Prx} Q$, At3g26060) and Glutathione Reductase 2 (EC 1.6.4.2, GR, At3g54660) genes as oxidative stress response markers (Table 2). The previously reported set of primers for Chloroplast Vesiculation gene ( $\mathrm{CV}$, At2g25625) were used to analyze $C V$ expression as a marker for chloroplast degradation (Wang and Blumwald 2014). The internal reference genes selected were the Arabidopsis housekeeping gene Actin-2 (ACT-2, At3g18780) and the Arabidopsis ubiquitin-conjugating enzyme gene (UBC, At5g25760). Both sets of primers were from Sigma (USA) and used according to the manufacturer's instructions.

Table 2. Primers used for real time PCR analysis. The gene identification corresponds to the sequence annotated in the TAIR database (The Arabidopsis Information Resource, https://www.arabidopsis.org/) and used as template for primer design. F: forward and R: reverse primers.

\begin{tabular}{llll}
\hline Gene Identification & TAIR Accession number & Primer Identification & Sequence (5'->3') \\
\hline Catalase 3 & At1g20620 & AtCAT3-F & GATCATGGGCACCAGACAGG \\
\hline L-ascorbate peroxidase 1 & At1g07890 & AtCAT3-R & CGGCTTGCAAGTTTCTGTCC \\
& & AtAPX1-F & CACCCATCATGGTCCGACTC \\
Peroxiredoxin $\mathbf{Q}$ & At3g26060 & AtAPX1-R & CTTGCTCAGCGTCAAACCTCA \\
& & AtPRXQ-F & GCTGGTGCAGAGGTCATTGG \\
Glutathione reductase $\mathbf{2}$ & At3g54660 & AtPRXQ-R & TCCAGGCACTCCCCAATCTT \\
& & AtGR2-F & GGGCTGTTGGGGATGTTACTG \\
\hline
\end{tabular}


The complementary DNA (cDNA) synthesis and amplification were performed using a SuperScript ${ }^{\circledR}$ III Platinum ${ }^{\circledR}$ SYBR ${ }^{\circledR}$ Green One-Step qRT-PCR Kit (Invitrogen by Life technologies, USA), according to the manufacturer's instructions. Thermal cycling conditions were set at 3 min hold at $50{ }^{\circ} \mathrm{C}$ for cDNA synthesis and $95{ }^{\circ} \mathrm{C}$ for $5 \mathrm{~min}$ hold, followed by 40 cycles consisting of $15 \mathrm{~s}$ at $95{ }^{\circ} \mathrm{C}, 45 \mathrm{~s}$ at $60{ }^{\circ} \mathrm{C}$ and $1 \mathrm{~min}$ at 40 ${ }^{\circ} \mathrm{C}$ in a Touch ${ }^{\mathrm{TM}}$ Real-Time PCR Detection System (Bio-Rad, model CFX96, USA). To test primer specificity, melting curve analysis (from $65{ }^{\circ} \mathrm{C}$ to $95{ }^{\circ} \mathrm{C}$ with an increasing heat rate of $0.5{ }^{\circ} \mathrm{C} \mathrm{s}^{-1}$ ) was performed following amplification and a notemplate control was also included. All experimental samples and the relative abundance values were calculated relative to the level of gene expression of the Time $0\left(\mathrm{~T}_{0}\right)$ sample as an internal control before induction, as well as to the housekeeping genes $A C T-2$ and $U B C$. Relative quantification of gene expression was carried out and the geometric averaging of multiple internal control genes (Vandesompele et al., 2002) was used to normalize the raw data.

\subsection{Genes and co-expression analysis of salt stress microarray}

In order to analyze the co-expression network of the four oxidative stress response gene markers evaluated by qRT-PCR (CAT3 At1g20620, APX1 At1g07890, Prx $Q$ At3g26060 and GR2 At3g54660), the experimental microarray AtGenExpress data previously reported for $A$. thaliana wild type (Col-0) under salt stress (Kilian et al., 2007) was studied using bioinformatic models. Experimental microarray data on shoot samples grown under normal (control plants, GSE5620) and salt stress (150 $\mathrm{mM} \mathrm{NaCl}$, GSE5623) conditions were obtained by analyzing the Gene Expression Omnibus (GEO) database (Barrett et al., 2011). Microarray data analysis was conducted as follows: public data was extracted and processed using the GEOquery package in Bioconductor. After individual microarrays analysis, different probes were mapped with the corresponding Entrez gene identification using the default platform file (GPL198). Genes with more than one probe were combined using the row with the highest mean intensity value applying the collapseRows and intersect functions implemented in the WGCNA package (Langfelder and Horvath 2008). This resulted in 60 samples (36 control and 24 salt stressed) and 21434 genes. Because the present work focused on four genes, we were particularly interested in the co-expression pattern of those genes. To accomplish this, we evaluated the Pearson's correlation coefficient between these four genes and the entire gene space of the microarray in both conditions. We fixed the correlation cutoff to 0.9 to reduce the network size to the most relevant co-expressions. The network was visualized using Cytoscape 3.4.0. The gene ontologies and metabolic pathways (KEGG database) enrichment analysis were performed using the DAVID Bioinformatics resource. The biological processes and metabolic pathways presented in the networks correspond to those with a $p$-value $<0.05$ in the enrichment analysis. The frequency of each significant biological process was calculated using REVIGO (Supek et al., 2011). The frequency of a biological process category is a measure of how recurrent this biological process is in the gene space; therefore, higher values correspond to less specific terms. 


\subsection{Determination of antioxidant enzymes activity, content and protein oxidative damage}

The evaluation of enzymatic activities was performed comparing equal amounts of total protein extracts from the samples collected at 24 and 72 hours as well as the control sample $\left(\mathrm{T}_{0}\right)$. CAT activity was measured by monitoring the decomposition of an $\mathrm{H}_{2} \mathrm{O}_{2}$ (hydrogen peroxide) solution at $240 \mathrm{~nm}$ as a consequence of the catalytic activity of the enzyme (Aebi 1984). Level of total glutathione (reduced glutathione (GSH) and glutathione disulfide (GSSG)) was determined using a Glutathione Assay kit (SigmaAldrich, USA) and peroxidase (POD) activity using an Amplex ${ }^{\circledR}$ Red Hydrogen Peroxide/Peroxidase Assay Kit (Invitrogen by Life technologies, USA), both following the manufacturer's instructions. Total carbonyl content of soluble components of the plant extracts was determined using dinitrophenyl hydrazine (DNPH) (Levine et al., 1990).

\subsection{Quantification of photosynthetic pigment content}

After collection, plant leaves were submerged in boiling water for 10 seconds to avoid the artificial conversion of $\mathrm{Chl}$ to chlorophyllide and immediately frozen in liquid nitrogen $\left(\mathrm{N}_{2}\right)$. The tissue was later ground in liquid $\mathrm{N}_{2}$ and $0.5 \mathrm{~mL}$ of $80 \%$ acetone was added and left overnight at 4 ${ }^{\circ} \mathrm{C}$ in the darkness. Tubes were centrifuged at 20 $000 \mathrm{x} \mathrm{g}$ for 5 minutes at $4{ }^{\circ} \mathrm{C}$, supernatant was collected and the absorbance at $663 \mathrm{~nm}$ and 645 $\mathrm{nm}$ was measured (BMG LABTECH GmbH, model FLUOstar Omega, Germany). Total Chl contents and Chl $a / b$ ratio were calculated as described elsewhere(Porra 2002).
Chl $b$ content was also determined by High-performance liquid chromatography (HPLC) analysis. To extract the pigment, $1 \mathrm{~mL}$ of HPLC grade methanol was added to $20 \mathrm{mg}$ of ground sample, and left at $4{ }^{\circ} \mathrm{C}$ for $16 \mathrm{~h}$. After this time, the mixture was centrifuged at $20000 \mathrm{x} \mathrm{g}$ for $5 \mathrm{~min}$ at $4{ }^{\circ} \mathrm{C}$ and the supernatant was used for direct injection in HPLC. Chl $b$ identification analyses were carried out using an Agilent Infinity 1200 series high performance liquid chromatograph (Agilent Technologies, 1260 Infinity, Waldbronn, Germany) with an Agilent 1260 Quaternary Pump, 1260 Infinity Standard Autosampler, and a diode array and multiple wavelength detector coupled to an HP ChemStation data-processing station. The column used was an Eclipse XDB-C18 (Agilent, 4.6 x150 $\mathrm{mm} ; 5 \mu \mathrm{m})$. The mobile phase consisted of an $\mathrm{H}_{2} \mathrm{O}-$ methanol mix (1:1, v:v) (eluent A) and methanol $100 \%$ (eluent $\mathrm{B}$ ). The gradient program used was as follows: $10 \% \mathrm{~A}$ and $90 \% \mathrm{~B}$ over $25 \mathrm{~min}, 0 \% \mathrm{~A}$ and $100 \% \mathrm{~B}$ over $5 \mathrm{~min}, 10 \% \mathrm{~A}$ and $90 \% \mathrm{~B}$ over $1 \mathrm{~min}$ and isocratic for $5 \mathrm{~min}$. The injection volume for all samples was $10 \mu \mathrm{L}$ and the flow rate was $0.5 \mathrm{~mL} \mathrm{~min}^{-1}$. Identification of $\mathrm{Chl} b$ was carried out by comparing retention time and spectral characteristics of unknown analytes with $\mathrm{Chl} b$ standard (Sigma Aldrich) using the HP ChemStation software (HP Hewlett-Packard ChemStation, Rev. C.01.04). Spectroscopic data from all peaks were accumulated in the range $410-445 \mathrm{~nm}$. Chl $b$ (0.0049- $0.0786 \mathrm{mM}$ ) was used for the calibration curve and results were expressed as $\mathrm{mM}$ of $\mathrm{Chl} b$ equivalents per dry mass (mg Chl $b$ per dry mass).

\subsection{Estimation of total phenolic content}

The Folin-Ciocalteu method was used to determine the total phenolic content (TPC) as reported 
by Singleton (Singleton et al., 1999)\{Singleton, $1999 \# 66\}$. Briefly, $100 \mu \mathrm{L}$ of samples, diluted $1: 10$ were mixed with $0.5 \mathrm{~mL}$ of $0.2 \mathrm{~N}$ Folin-Ciocalteu reagent for $5 \mathrm{~min}$ and $0.4 \mathrm{~mL}$ of $0.7 \mathrm{M}$ sodium carbonate $\left(\mathrm{Na}_{2} \mathrm{CO}_{3}\right)$. After incubation in the dark at $25{ }^{\circ} \mathrm{C}$ for $2 \mathrm{~h}$, the absorbance of the reaction mixture was measured at $760 \mathrm{~nm}$ using a FLUOstar Omega Microplate Reader (BMG LABTECH $\mathrm{GmbH}$, Germany). Gallic acid was used as the standard to produce a calibration curve $(0.5-1.5$ $\mathrm{mM})$. The TPC was expressed in $\mathrm{mg}$ of gallic acid equivalents (mg GAE per mg of dry weight).

\subsection{Statistical analysis}

For all variables analyzed, each experiment was performed in triplicate. The data were expressed as means and evaluated by analysis of variance (ANOVA) using SPSS Statistics (Version 24.0), and compared using Duncan's range test at $p=$ 0.05 . All the results are shown as an average \pm standard deviation (SD) of the three independent replicates.

\section{Results}

\subsection{Gene expression}

To examine the effect of XGOs on A. thaliana plants grown under saline conditions, gene expres- sion of oxidative stress markers was measured in leaves after exposure to salt shock, XGOs or a combination of both for 24 and $72 \mathrm{~h}$ (Figure 1). Expression of two genes was significantly altered by the treatments: CAT2,3 and Prx Q. CAT2,3 was up-regulated by 2.45 -fold with respect to the untreated control at $24 \mathrm{~h}$ by XGOs alone. However, the most dramatic positive effect was with the combination of XGOs and salt shock where an increase of 4.8-fold was elicited compared to the MS control at $24 \mathrm{~h}$ after induction. There was also an apparent increase in CAT2,3 gene expression in the salt+XGO treatment, compared to salt alone, although differences were not statistically significant. After $72 \mathrm{~h}$, the effects on CAT2,3 gene expression were different: neither salt alone or in combination with XGOs affected expression, but XGOs alone increased expression 2.85-fold compared to the MS control (Figure 1A). Prx $Q$ was up-regulated by 1.54 -fold with respect to the untreated control at $24 \mathrm{~h}$ by XGOs alone, but after 72 $\mathrm{h}$ the expression level decreased to even below the control (Figure 1B). Prx $Q$ expression was significantly lower under salt stress compared to the MS control at both time points. However, no differences were observed with salt $+\mathrm{XGO}$ treatment at either 24 or $72 \mathrm{~h}$ after induction, compared to salt stress alone. Gene expression of APX1 and GR2 did not show statistically significant differences amongst the treatments (Figure $1 \mathrm{C}-\mathrm{D}$ ). 

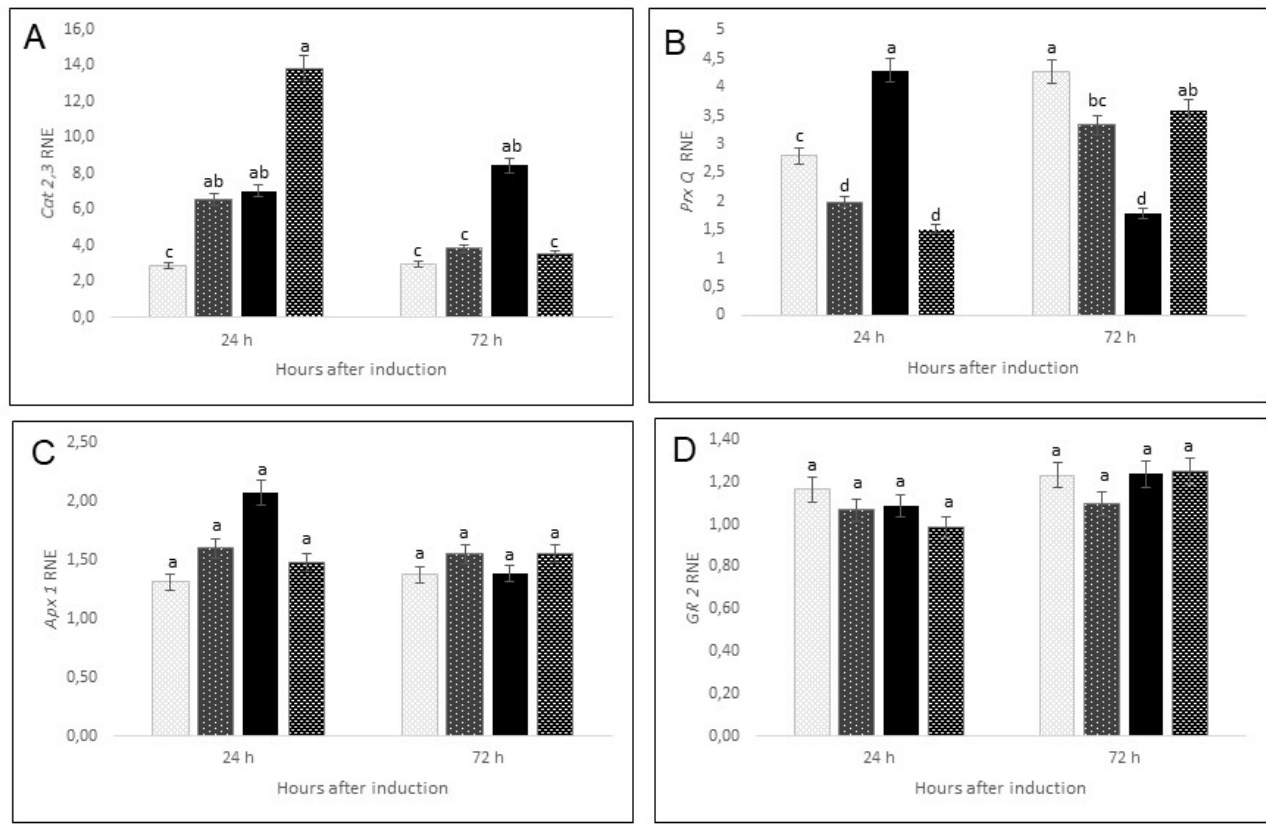

MS $\mathrm{MS}+\mathrm{NaCl}$

XGO 舜 $\mathrm{XGO}+\mathrm{NaCl}$

Figure 1. Expression levels by qRT-PCR, and protein level analysis of oxidative stress markers in Arabidopsis thaliana leaves $\pm 100 \mathrm{mM}\left(10 \mathrm{dSm}^{-1}\right) \mathrm{NaCl}, \pm 0.1 \mathrm{mg} \mathrm{L}^{-1} \mathrm{XGO}$, or a combination of both, at 24 and 72 hours after induction. Quantitative RT-PCR of genes (A) CAT2,3 (At1g20620), (B) APX1 (At1g07890), (C) GR2 (At3g54660) and (D) $\operatorname{Prx} Q$ (At3g26060). Mean \pm SD values were obtained from three independent experiments and normalized to the levels of two internal controls, Actin-2 (ACT-2, At3g18780) and Ubiquitin-Conjugating enzyme gene (UBC, At5g25760). Experiments were repeated three times with similar results. All the analyses were relative to the Time $0\left(\mathrm{~T}_{0}\right)$ sample as a control before induction and different letters indicate statistically different means $p<0.05$. RNE means Relative Normalized Expression.

CAT and POD (representing peroxidases) antioxidant enzyme activities were analyzed by spectrophotometry (Figure 2). CAT showed a significant reduction in its activity following salt shock, compared to the MS control. However, addition of XGOs promoted a significantly higher CAT activity with respect to the untreated control $24 \mathrm{~h}$ after induction. After $72 \mathrm{~h}$ the increase appeared to be sustained, but was no longer statistically significant. XGOs combined with saline treatment also elevated CAT activity significantly compared to salinity alone at both time points, but activity was not significantly higher than XGOs alone at either time point (Figure 2A). XGOs also induced a significant increase in POD activity compared to the untreated control, at 24 and $72 \mathrm{~h}$ after induction. However, no statistical differences were observed when XGOs were applied together with the salt compared to the $\mathrm{NaCl}$ alone control (Figure 2B). 

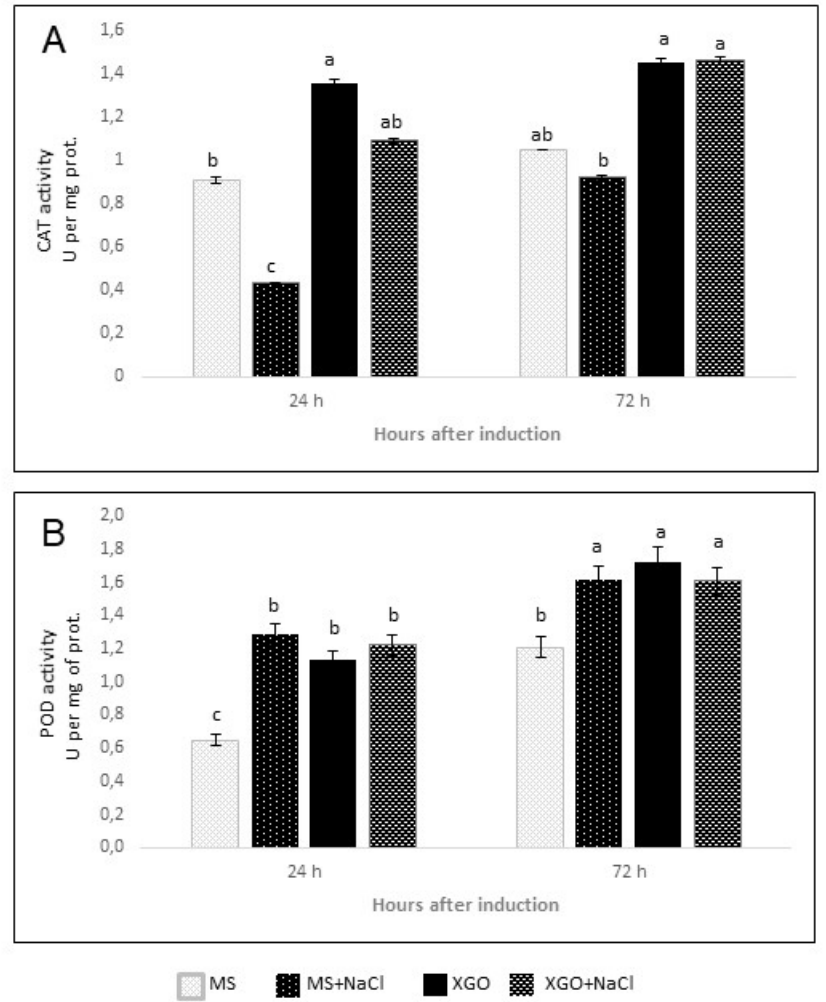

Figure 2. Enzymatic activity analysis in Arabidopsis thaliana leaves $\pm 0.1 \mathrm{mg} \mathrm{L}^{-1} \mathrm{XGO}, \pm 100 \mathrm{mM}\left(10 \mathrm{dSm}^{-1}\right)$ $\mathrm{NaCl}$ or a combination of both. Total proteins from leaves at 24 and 72 hours after induction were obtained and analyzed by spectrophotometric analyses. (A) CAT activity, (B) POD activity. Mean \pm SD values were obtained from three independent experiments. All the analyses were relative to a Time $0\left(\mathrm{~T}_{0}\right)$ sample as a control before induction and different letters indicate statistically different means $p<0.05$.

\subsection{Photosynthetic pigments content and chloroplast degradation}

Exposure to saline conditions significantly decreased the $\mathrm{Chl} \mathrm{a} / b$ ratio (Figure $3 \mathrm{~A}$ ) and $\mathrm{Chl} b$ content (Figure $3 \mathrm{~B}$ ) in the leaves of $A$. thaliana after $72 \mathrm{~h}$. However, XGOs alone promoted a significant increase of $\mathrm{Chl} \mathrm{a} / b$ ratio but not $\mathrm{Chl} b$ content after $72 \mathrm{~h}$ in comparison with the untreated control. Furthermore, XGOs in combination with salt were able to amelio- rate the drop in $\mathrm{Chl} \mathrm{a} / b$ ratio and $\mathrm{Chl} b$ content elicited by the salt treatment $72 \mathrm{~h}$ after induction. In contrast expression of $C V$ was not up-regulated by either salt or XGOs treatment at $24 \mathrm{~h}$, in fact there was a slight but not statistically significant reduction in $C V$ expression with XGO treatment. However, after $72 \mathrm{~h}$ of salt treatment $C V$ expression was up-regulated by 2.61-fold with respect to the untreated control. Its expression was not affected by the treatments with XGO alone or salt $+\mathrm{XGO}$ although there was a slight, but 
not statistically significant rise in expression following salt treatment (Figure 3C). There was also signifi- cantly less expression of $\mathrm{CV}$ in the $\mathrm{XGO}+\mathrm{NaCl}$ treatment compared to the $\mathrm{NaCl}$ treatment alone after $72 \mathrm{~h}$.
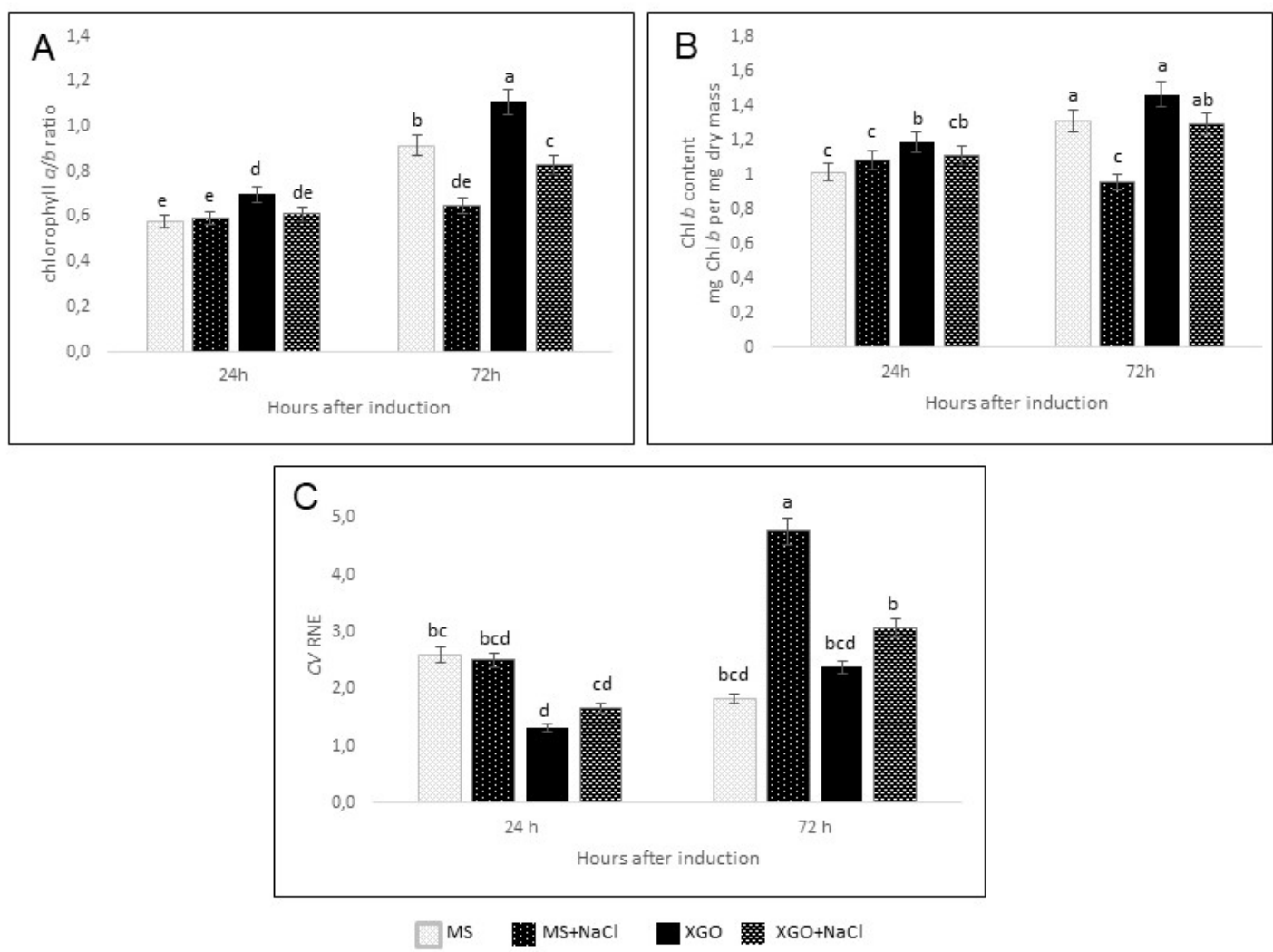

Figure 3. Chlorophyll content and chloroplast vesiculation gene $(\mathrm{CV})$ analysis in Arabidopsis thaliana leaves \pm $0.1 \mathrm{mg} \mathrm{L}^{-1} \mathrm{XGO}, \pm 100 \mathrm{mM}\left(10 \mathrm{dSm}^{-1}\right) \mathrm{NaCl}$ or a combination of both. Mean $\pm \mathrm{SD}$ values were obtained from three independent experiments; different letters indicate statistically different means $p<0.05$. All the analyses were relative to a Time $0\left(\mathrm{~T}_{0}\right)$ sample as a control before induction. (A) $\mathrm{Chl}$ a/ $b$ ratio determined by spectrophotometric analysis, (B) Chl $b$ content determined by HPLC analysis expressed in mg Chl $b$ per dry mass, (C) qRT-PCR analysis of $C V$ gene (At2g25625) expression. RNE means Relative Normalized Expression.

\subsection{Bioinformatic analysis}

There are no data available from studies of gene expression or microarray analysis in leaf samples treated with XGOs under saline conditions in $A$. thaliana. However, we analyzed available information from saline stress data previously obtained from global transcriptomic studies in Arabidopsis (Kilian et al., 2007), although it was only available for shoots and not mature leaves. The coexpression network obtained showed that under normal growing conditions (control plants) there was no direct correlation amongst the expression patterns of the four genes analyzed here (CAT, 
$A P X, \operatorname{Prx} Q$ and $G R$ ), and each one formed an independent group at the predefined cutoff (Figure 6). However, under saline stress conditions a set of genes act as mediators between CAT and Prx $Q$ genes. In both networks (control or salt shock) the $A P X$ gene remains unconnected and the coexpression around the $G R$ gene persists as an independent cluster (Figure 7). The greatest modification in the co-expression pattern was around the $\operatorname{Prx} Q$ gene, as it showed the major connectivity increment compared to normal conditions. Among the 28 genes acting as mediators between $C A T 3$ and Prx $Q$ genes, At3g52070, At3g19900 and At5g01790 have no function assigned to them. Three are genes related to abiotic or biotic stress responses as ultraviolet-B receptors with UV-protective functions; the $C A M B P 25$ gene encodes a $25 \mathrm{kDa}$ calmodulin (CAM)-binding protein and is induced by dehydration and ionic (salt) and nonionic (mannitol) osmotic stress, and MLP43, is a gene which encodes MLP-like protein 43 that is induced under drought stress. Also of relevance is a gene involved in oxidation-reduction processes, nudix hydrolase 7 (NUDT7) which encodes a protein with ADP-ribose hydrolase activity and regulates defense mechanisms against oxidative DNA damage. In addition, there are genes involved in chloroplast function that allow the plant to modify its photosynthetic capacity and to adapt to environmental changes and stresses, comprising two thioredoxin genes (ATHM2 and TH7) and two ferredoxin genes (At2g04700 and FNR2).

Under saline stress conditions, in the biological processes network, a set of processes act as mediators between $C A T 3, G R 2$ and $\operatorname{Pr} x Q$ gene expression (Figure 4A). Nevertheless, genes related to oxidation-reduction processes which connect GR2 with CAT3 and Prx $Q$ appear to be for very global and general biological processes according to the frequency values. When the relationships between these genes and their significantly enriched metabolic pathways was investigated, the co-expression network around the $G R$ gene remained as an independent cluster (Figure 4B). In agreement with this, in our experimental results we did not find any differences in GR2 gene expression in response to salt shock (Figure 1D). 
A

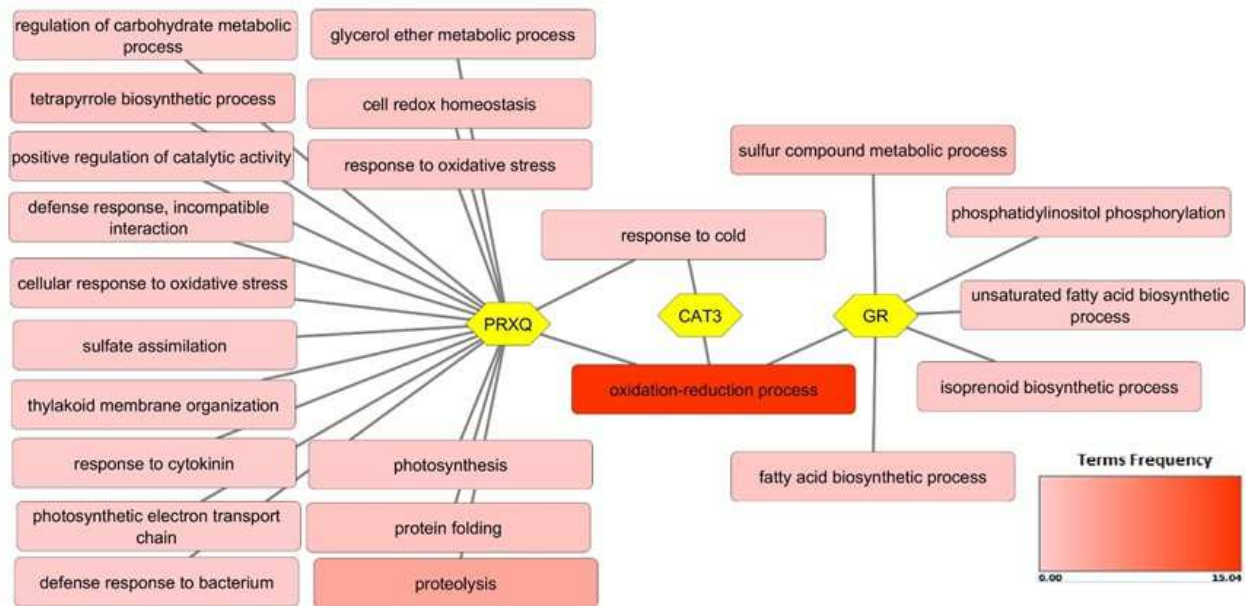

B
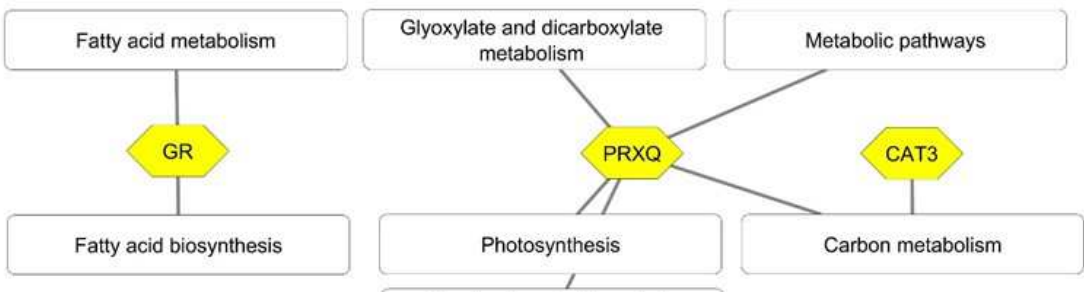

Porphyrin and chlorophyll metabolism

Figure 4. Co-expression network between catalase 3 (CAT3), L-ascorbate peroxidase 1 (APX), peroxiredoxin $Q$ $(\operatorname{Prx} Q)$ and glutathione reductase 2 (GR) genes under saline stress and their significantly $(\mathrm{p}<0.05)$ enriched: (A) biological processes and (B) metabolic pathways. Hexagons are genes, rectangles are biological processes and the color scale shows the frequency distribution of each biological process.

\subsection{Oxidative damage, protein oxidation and total} polyphenol content

The effect of XGOs on glutathione content as a marker for protection against oxidative stress was also determined. Here, the $A$. thaliana plants grown under saline conditions, with or without XGOs, did not show significant changes in total glutathione (GSH and GSSG) level amongst any of the treatments evaluated (Figure $5 \mathrm{~A}$ ). This agrees with the gene expression results, where no significant variation of GR2 gene expression was observed in any of the treatments (Figure 1C, E). 


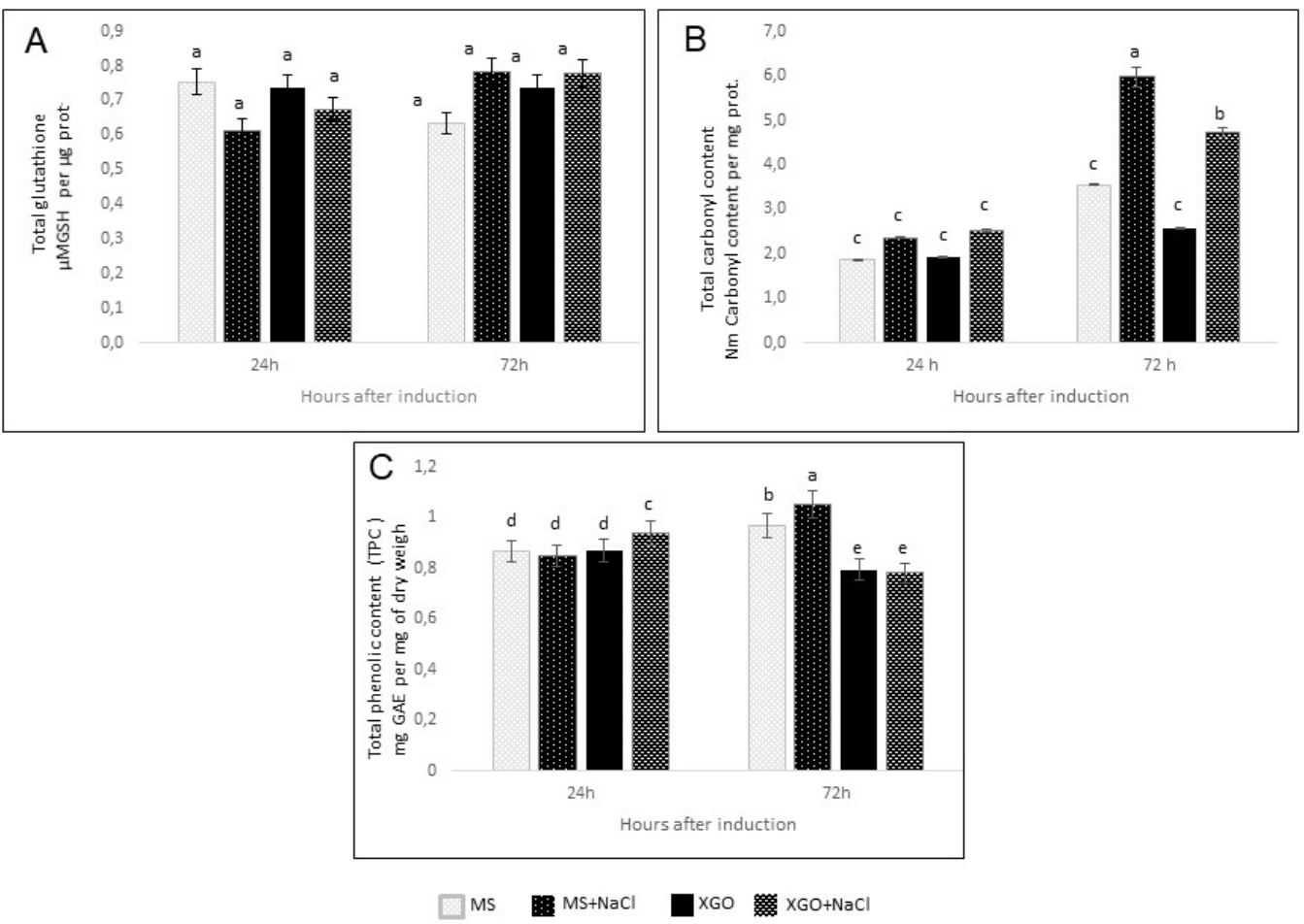

Figure 5. Analysis of markers of antioxidant metabolites in Arabidopsis thaliana leaves $\pm 0.1 \mathrm{mg} \mathrm{L}^{-1} \mathrm{XGO}, \pm$ $100 \mathrm{mM}\left(10 \mathrm{dSm}^{-1}\right) \mathrm{NaCl}$ or a combination of both. Mean $\pm \mathrm{SD}$ values were obtained from three independent experiments; different letters indicate statistically different means $p<0.05$. All the analyses were relative to a Time $0\left(\mathrm{~T}_{0}\right)$ sample as a control before induction. (A) Level of total glutathione (reduced glutathione (GSH) and glutathione disulfide (GSSG)) expressed as $\mu \mathrm{M}$ GSH per $\mu$ g of protein, (B) Total carbonyl content expressed as nm of carbonyl content per mg of protein, (C) Total phenolic content (TPC) expressed in mg of gallic acid equivalents (mg GAE per mg of dry weight).

In order to analyze the effect of XGOs on oxidative damage, protein carbonyl content was evaluated as a marker for protein oxidation. At $24 \mathrm{~h}$ after induction, no statistically significant differences were observed with any of the treatments, but after $72 \mathrm{~h}$ the salt treatment alone induced a significant accumulation of protein carbonyls relative to the untreated control. In contrast, addition of XGOs promoted a significant reduction of these compounds. In the presence of salt, protein carbonyl levels appeared lower, but were not statistically different from the control treatment (Figure 5B), however the combination of $\mathrm{XGO}+\mathrm{NaCl}$ resulted in reduced carbonyl content compared to salt alone. The salinity treatment for $72 \mathrm{~h}$ also significantly increased total phenolics (Figure 5C). However, the addition of XGOs significantly reduced these levels, even in the presence of $\mathrm{NaCl}$. 

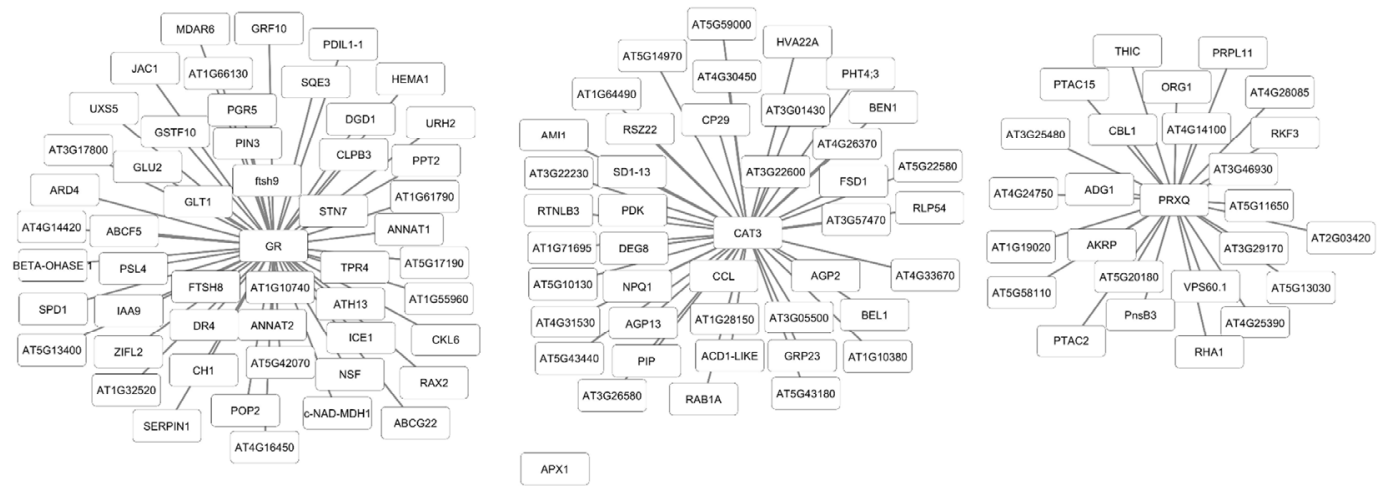

Figure 6. Co-expression network of catalase 3 (CAT3), L-ascorbate peroxidase 1 (APX1), peroxiredoxin Q (Prx $Q$ ) and glutathione reductase 2 (GR2) gene using the experimental microarray for Arabidopsis thaliana Wild Type (col-0) under normal conditions.

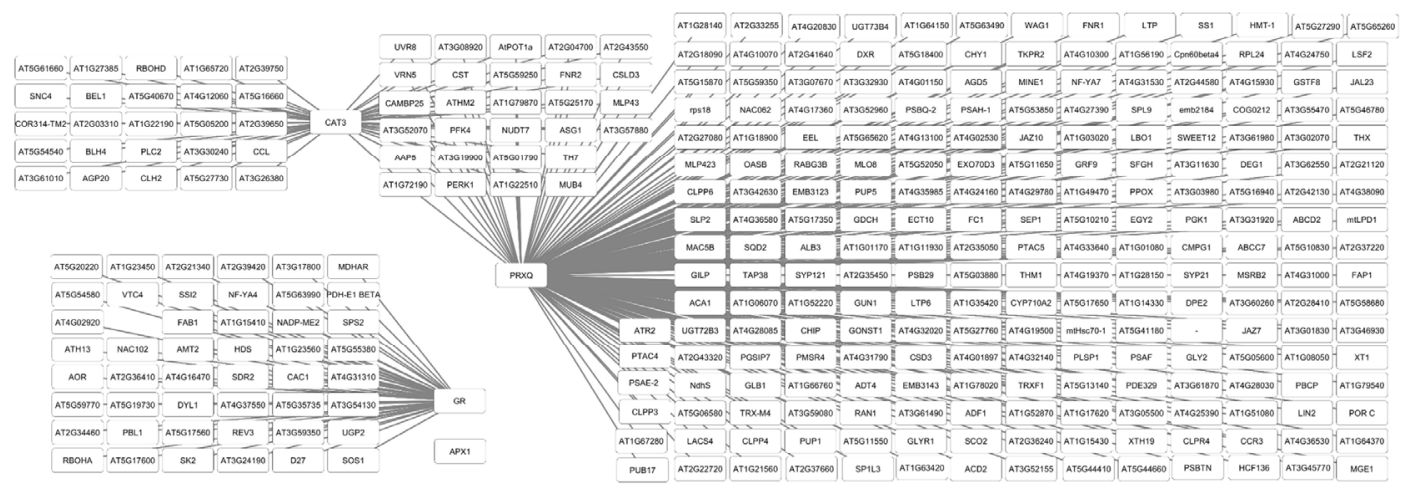

Figure 7. Co-expression network of catalase 3 (CAT3), L-ascorbate peroxidase (APX1), peroxiredoxin $Q$ (Prx $Q$ ) and glutathione reductase (GR2) gene using the experimental microarray for Arabidopsis thaliana Wild Type (col-0) under salt stress.

\section{Discussion}

Detoxification of ROS is an important component of a plant's protection against salt stress, but efficient antioxidative defense does not necessarily mean the strong up-regulation of the full set of antioxidant enzymes. Thus, antioxidant enzymes (CAT, POD, APX and GR) can have a contrasting pattern of expression since they have different functions in the cell. Furthermore, the relative importance of each enzyme required for ROS scavenging varies from plant to plant, according to the rates of ROS generation and the major source of ROS (Gill and Tuteja 2010). Here the results indicated that the role of XGOs in relation to ROS scavenging was limited to stimulating an increase in $C A T$ gene expression and CAT and POD enzyme activity. 
The chlorophyll content reduction is another consequence of salt stress in plants (Taïbi et al., 2016; Hamdani et al., 2017). Degradation of energy-absorbing pigments can be a defense mechanism against ROS, because ROS production is driven mainly by excess energy absorption by the photosynthetic apparatus. Chl $b$ degradation in plants promotes nutrient remobilization, participates in the suppression of photodamage and regulates the plant light-harvesting capacity (Christ and Hörtensteiner 2014). Thus, Chl content is widely used as an index of abiotic tolerance in plants. Consequently, it would seem that XGOs may help to reduce $\mathrm{Chl}$ breakdown under salt stress and this may contribute to a protective effect of the $\mathrm{XGOs}$ to increase tolerance to saline stress conditions. XGOs may do this by a reduction in chloroplast degradation via the mechanism that includes the action of the $C V$ gene. In agreement with this idea, Wang and Blumwald (2014) proved that $C V$ silencing in Arabidopsis resulted in chloroplast stability during stress and enhanced stress tolerance. The down-regulation of $C V$ expression was also observed in rice (Oryza sativa) plants under water stress, leading to water-deficit stress tolerance (Sade et al., 2018). However, it is not possible to conclude whether down-regulation of $\mathrm{CV}$ expression is a direct effect of the XGOs. Prx Q protein level is also particularly important due its location in the lumen of the thylakoid membrane of A. thaliana and its function in oxidant detoxification and redox regulated signaling in chloroplasts (Petersson et al., 2006), so it can impact directly on photosynthesis. In A. thaliana there was a reduction in chlorophyll when the $\operatorname{Prx} Q$ gene was knocked out, which suggested its role in protection of photosynthetic enzymes (Lamkemeyer et al., 2006). This suggests that the up-regulation of the $\operatorname{Prx} Q$ gene expression observed at 24 $\mathrm{h}$ after XGO application might indicate a mechanism that protects chloroplasts from degradation. Our findings thus indicate that XGOs are able to mitigate the effects of salt stress on chlorophyll levels, which is reinforced by the $\operatorname{Prx} Q$ gene up-regulation observed. The reduction in Chl degradation may be also a consequence of the activation of antioxidant mechanisms, including increased CAT and POD enzyme activity. The increase in both enzyme activities of plants treated with XGOs combined with salt stress is in agreement with previous results on wheat seedlings (Triticum aestivum L.) with low-molecular-weight polysaccharides (Zou et al., 2018). In addition, it has been reported that CAT is one of the most effective antioxidant enzymes in preventing cell damage (Mhamdi et al., 2010). Our data showed that in saline conditions, catalase gene expression and enzymatic activity was dramatically increased by XGOs. This suggests that $\mathrm{XGO}$ may be contributing to plant protection against salt-induced oxidative damage through an elevated antioxidant capacity.

We also examined the four oxidative stress response gene markers in the context of gene co-expression networks from microarray data of Arabidopsis under salt stress. Our findings suggest that the strongest relationship is between $\operatorname{Prx} Q$ and CAT3 with processes like photosynthesis under salt shock compared to normal conditions, which is in agreement with our experimental results. Besides, GR2 expression under salt stress is highly connected with lipid metabolism and only connected with $\operatorname{Prx} Q$ or $C A T 3$ through oxidation-reduction processes, which are very general processes. Under stress, GR2 expression could be regulated by lipid metabolism, however, we did not evaluate any markers related with lipid profiles. Moreover, the carbon metabolism category, which is connecting CAT3 and $\operatorname{Prx} Q$ genes, includes the Calvin cycle. More experiments are needed in order to confirm whether the identified intermediary genes are altered by XGO treatment under salt shock. This analysis of the connectivity amongst the ROS-related genes under salt stress, provides useful pointers 
for further analyses of gene expression and metabolic processes, to test whether they are affected by the combination of XGOs and salt stress. This might then help to unravel the mechanism of action of the XGOs in reducing effects of salt stress on the photosynthetic apparatus.

Another consequence of high intracellular ROS level is the oxidation of vital biomolecules, such as proteins and DNA, and cells undergo oxidative damage. The effect of XGOs on reduction of protein damage could help the plant to prevent or tolerate oxidative stress. Salinity stress also stimulates the synthesis and accumulation of polyphenols, which is probably related with an increase in secondary metabolites, that protect the plant against ROS (Valifard et al., 2014). In this study, the phenolic content with the addition of XGOs alone or combined with salt shock was significantly reduced.

XGOs may be acting through modifications or integration into the cell wall, which can affect not only the extracellular events in the wall but also intracellular events (Takeda et al., 2002). When pea stem segments partially bisected longitudinally were incubated with a xyloglucan oligosaccharide ( $9 \mathrm{mM} \mathrm{XXXG)}$, cell elongation was accelerated by integration of xyloglucans as they were incorporated into the cell wall and became transglycosylated by xyloglucan endotransglycosylase (XET). According to this, the effect of XGOs observed in our work may result not from a direct action of the oligosaccharide but instead from a signal transduction cascade mediated by XET or induced by the cell wall modification.

\section{Conclusions}

Overall, we conclude that exogenous application of XGO can exert beneficial impacts on the response of Arabidopsis thaliana seedlings to salt stress. This may be mediated by increased expression and activity of
ROS-related enzymes, such as catalase, as the primary mechanism of their action. XGO treatment results in improved photosynthetic efficiency and increased non-enzymatic ROS-scavenging metabolites, while reducing protein oxidation and total polyphenols. As far as we know, this is the first report that analyses the effect of XGOs on salt stress. Further in vivo studies are needed to confirm the antioxidant effect of XGOs during salt stress both in model and crop plants as well as to unravel their mechanism of action on oxidative responses. However, in the light of these results, the application of low concentrations of XGO could be considered as a biostimulant for improving the growth and productivity of crops of agronomic importance under salt stress.

\section{Acknowledgments}

This study was funded by Universidad de Las Américas (UDLA), Quito, Ecuador.

\section{References}

Abogadallah, G.M. 2010. Antioxidative defense under salt stress. Plant Signaling and Behavior. 5, 369-74.

Aebi, H. 1984. Catalase in vitro. in, Methods in Enzymology (Academic Press).

Barrett, T., Troup, D.B., Wilhite, S.E., Ledoux, Pierre., Evangelista, Carlos., Kim, I.F., Tomashevsky, M., Marshall, K.A., Phillippy, K.H., Sherman, P.M., Muertter, R.N., Holko, M., Ayanbule, O., Yefanov, Andrey., Soboleva, A. 2011. NCBI GEO: archive for functional genomics data sets-10 years on. Nucleic Acids Research. 39, D1005-D10.

Bulgari, R., Cocetta, G., Trivellini, A., Vernieri, P., Ferrante, A. 2015. Biostimulants and crop responses: a review. Biological Agriculture and Horticulture. 31, 1-17. 
Cabrera, J.C., Wégria, G., Onderwater, R.C.A., Napoles, M.C., Falcon-Rodriguez, A.B.D., Diosdado, E., Gonzalez, S., González, G. Gonzalez, L., Rogers, H.J., Cabrera, G., Wattiez, R. 2012. Practical use of oligosaccharins in agriculture. Acta Horticulturae. 1009, 195-212.

Bastien, C., Hörtensteiner, Stefan. 2014. Mechanism and significance of chlorophyll breakdown. Journal of Plant Growth Regulation. 33, 4-20.

Cutillas-Iturralde, A., Peña, M.J., Zarra, I., Lorences, E.P. 1998. A xyloglucan from persimmon fruit cell walls. Phytochemistry. 48, 607-610.

Fry, S.C., York, W.S., Albersheim, P., Darvill, A., Hayashi, T., Joseleau, J.P., Kato, Y., Pérez Lorences, E., Maclachlan, G.A., McNeil, M., Mort, A.J., Grant Reid, J.S., Ulrich Seitz, H., Selvendran, R.R., Voragen, A.G.J., White, A.R. 1993. An unambiguous nomenclature for xyloglucan-derived oligosaccharides. Physiologia Plantarum. 89, 1-3.

Gill, S.S., Tuteja, N. 2010. Reactive oxygen species and antioxidant machinery in abiotic stress tolerance in crop plants. Plant Physiology and Biochemistry. 48, 909-930.

González-Pérez, L., Perrotta, L., Acosta, A., Orellana, E., Spadafora, N., Bruno, L., Bitonti, B.M., Albani, D., Cabrera, J.C., Francis, D., Rogers, H.J. 2014. In tobacco BY-2 cells xyloglucan oligosaccharides alter the expression of genes involved in cell wall metabolism, signalling, stress responses, cell division and transcriptional control. Molecular Biology Reports. 41, 6803-16.

González-Pérez, L., Vázquez-Glaría, A., Perrotta, L., Acosta, A., Scriven, S.A., Herbert, R., Cabrera, J.C., Francis, D., Rogers, H.J. 2012. Oligosac-

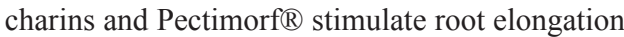
and shorten the cell cycle in higher plants. Plant Growth Regulation. 68, 211-21.
Hamdani, F., Derridj, A., Rogers, H.J. 2017. Diverse salinity responses in Crithmum maritimum tissues at different salinities over time. Journal of Soil Science and Plant Nutrition. 17, 716-34.

Kilian, J., Whitehead, D., Horak, J., Wanke, D., Weinl, S., Batistic, O., D’Angelo, C., BornbergBauer, E., Kudla, Jörg., Harter, Klaus. 2007. The AtGenExpress global stress expression data set: protocols, evaluation and model data analysis of UV-B light, drought and cold stress responses. The Plant Journal. 50, 347-63.

Kozioł, A., Cybulska, J., Pieczywek, P.M., Zdunek, A. 2015. Evaluation of structure and assembly of xyloglucan from tamarind seed (Tamarindus indica L.) with atomic force microscopy. Food Biophysics. 10, 396-402.

Lamkemeyer, P., Laxa, M., Collin, V., Li, W., Finkemeier, I., Schöttler, M.A., Holtkamp, V., Tognetti, V.B., Issakidis-Bourguet, E., Kandlbinder, A., Weis, E., Miginiac-Maslow, M., Dietz, K.J. 2006. Peroxiredoxin Q of Arabidopsis thaliana is attached to the thylakoids and functions in context of photosynthesis. The Plant Journal. 45, 968-81.

Langfelder, P., Horvath, Steve. 2008. WGCNA: an R package for weighted correlation network analysis. BMC Bioinformatics. 9, 559.

Levine, R.L., Garland, D., Oliver, C.N., Amici, A., Climent, I.A., Lenz, G., Ahn, B.W., Shaltiel, S., Stadtman, E.R. 1990. Determination of carbonyl content in oxidatively modified proteins. Methods in Enzymology (Academic Press).

Sutapa, M., Lerouge, P., Loutelier-Bourhis, C., Driouich, A., Ray, Bimalendu. 2005. Structural characterisation of hemicellulosic polysaccharides from Benincasa hispida using specific enzyme hydrolysis, ion exchange chromatography and 
MALDI-TOF mass spectroscopy. Carbohydrate Polymers. 59, 231-38.

Mhamdi, Amna, Queval, G., Chaouch, S., Vanderauwera, S., Van Breusegem, F., Noctor, Graham. 2010. Catalase function in plants: a focus on Arabidopsis mutants as stress-mimic models. Journal of Experimental Botany. 61, 4197-41220.

Murashige, T., and F. Skoog. 1962. A revised medium for rapid growth and bioassays with tobacco tissue cultures, Physiol Plant. 15, 473 - 97.

Petersson, Ulrika A., Thomas Kieselbach, José G. García-Cerdán, and Wolfgang P. Schröder. 2006. The Prx Q protein of Arabidopsis thaliana is a member of the luminal chloroplast proteome, FEBS letters. 580, 6055-61.

Porra, Robert J. 2002. The chequered history of the development and use of simultaneous equations for the accurate determination of chlorophylls a and b. Photosynthesis Research. 73, 149-56.

Sade, Nir., Kamolchanok, Umnajkitikorn., Rubio Wilhelmi, Maria Del Mar., Wright, Matthew., Wang, Songhu., Blumwald, Eduardo. 2018. Delaying chloroplast turnover increases water-deficit stress tolerance through the enhancement of nitrogen assimilation in rice. Journal of Experimental Botany. 69, 867-78.

Shavrukov, Yuri. 2013. Salt stress or salt shock: which genes are we studying? Journal of Experimental Botany. 64, 119-27.

Singleton, Vernon L., Rudolf, Orthofer., LamuelaRaventós, Rosa M. 1999. Analysis of total phenols and other oxidation substrates and antioxidants by means of folin-ciocalteu reagent. in, Methods in Enzymology (Academic Press).

Supek, Fran., Bošnjak, Matko., Škunca, Nives., Šmuc, Tomislav. 2011. REVIGO summarizes and visualizes long lists of gene ontology terms. PLoS ONE. 6, e21800.
Khaled, Taïbi., Fadhila, Taïbi., Ait Abderrahim, Leila., Ennajah, Amel., Belkhodja, Moulay., Mulet, José Miguel. 2016. Effect of salt stress on growth, chlorophyll content, lipid peroxidation and antioxidant defence systems in Phaseolus vulgaris L. South African Journal of Botany. 105, 306-312.

Takeda, Takumi., Furuta, Yuzo., Awano, Tatsuya., Mizuno, Koichi., Mitsuishi, Yasushi., Hayashi, Takahisa. 2002. Suppression and acceleration of cell elongation by integration of xyloglucans in pea stem segments. Proceedings of the National Academy of Sciences. 99, 9055-9060.

Valifard, M., Mohsenzadeh, S., Kholdebarin, B., Rowshan, V. 2014. Effects of salt stress on volatile compounds, total phenolic content and antioxidant activities of Salvia mirzayanii. South African Journal of Botany. 93, 92-97.

Vandesompele, Jo., De Preter, Katleen., Pattyn, Filip., Poppe, Bruce., Van Roy, Nadine., De Paepe, Anne., Speleman, Frank. 2002. Accurate normalization of real-time quantitative RT-PCR data by geometric averaging of multiple internal control genes. Genome Biology. 3, research0034.1

Wang, Songhu., Blumwald, Eduardo. 2014. Stressinduced chloroplast degradation in Arabidopsis is regulated via a process independent of autophagy and senescence-associated vacuoles. The Plant Cell. 26, 4875-4888.

You, Jun., Zhulong, Chan. 2015. ROS Regulation during abiotic stress responses in crop Plants. Frontiers in Plant Science. 6, 1092.

Zou, Ping., Xueli, Lu,, Changliang, Jing., Yuan, Yuan., Yi, Lu., Chengsheng, Zhang., Lei, Meng., Hongtao, Zhao., Yiqiang, Li. 2018. Low-molecularweightt polysaccharides from Pyropia yezoensis enhance tolerance of wheat seedlings (Triticum aestivum L.) to salt stress. Frontiers in Plant Science. 9 . 